\title{
Innovation and Development of Rural Leisure Tourism Industry Using Mobile Cloud IoT Computing
}

\author{
Guangwei Wang $(\mathbb{D}$ \\ Aviation and Tourism College, Guilin University of Aerospace Technology, Guangxi, China \\ Correspondence should be addressed to Guangwei Wang; wangguangwei198809@guat.edu.cn
}

Received 6 May 2021; Revised 26 July 2021; Accepted 11 August 2021; Published 26 August 2021

Academic Editor: Michel Kadoch

Copyright (c) 2021 Guangwei Wang. This is an open access article distributed under the Creative Commons Attribution License, which permits unrestricted use, distribution, and reproduction in any medium, provided the original work is properly cited.

\begin{abstract}
As a promising IoT application, the rural leisure tourism industry can promote the reconstruction of industrial structure in rural areas and realize a sustainable, rapid, and healthy development of rural economy. This paper takes the rural leisure tourism industry in China as an example and aims at building an intelligent and integrated modern IoT use case. Based on the traditional rural leisure tourism, we improve the system by adding the data analysis over a mobile cloud IoT computing platform. In particular, this work investigates the characteristics of the national tourism market under the security requirements from governmental cloud data management policy. Our study shows that the geographical concentration index $G$ of tourists in the Chinese market continues to increase. With the booming of IoT applications in rural leisure tourism, intelligent and integrated tourism guidance and optimized decision-making will provide tourists with better information and thus make rapid improvement of geographical concentration index.
\end{abstract}

\section{Introduction}

Modern rural leisure tourism is a new type of industry that uses agricultural production and rural ecological processes to provide consumers with entertainment, sightseeing, and experience services. Historical data shows that it has also promoted the transformation and upgrading of the agricultural industry. The Internet of Things (IoT) is a key supporting technology for rural leisure tourism. For developed countries, leisure agriculture and rural tourism are the consequences of urban industrial pollution and fast-paced lifestyles. As early as the 1830s, rural agricultural tourism began in Europe. With the continuous spread of global epidemics in the past two years, the rural leisure tourism has shown new vitality and become one of the important directions of the global tourism industry.

Information technology-based tourism has been widely studied in the past. By using the anonymous mobile location information of national mobile network operator of Estonia, Chen conducted an empirical analysis on the field visit data of foreign tourists based on the dimensions of geography, time, and composition [1]. Dai, instead, utilized big data technology over detailed telephone record (CDR) data and real-time location information of mobile phones, in order to monitor the flow of tourists in the scenic spot and analyze the tourist behavior in the scenic spot [2]. Based on big data, Zhou analyzed the influencing variables of the branding of tourism destinations on Facebook [3]. Qi used social media data from Flickr and Twitter to assess the potential tourism security threats to bird and biodiversity areas that are globally well-known and believes that the development of tourism can promote the protection of biodiversity areas [4]. Zhou conducted social network and semantic big data analysis on tourist content retrieved from online communities in the TripAdvisor forums in 7 major European capital cities and conducted a tourism demand forecast analysis [5].

In the meantime, Porambage made use of Barcelona as an example to analyze the influence of social networks on Chinese tourists' travel behavior [6]. Smith collected real-time big data of Wi-Fi routers installed in 149 different locations on Jeju Island, South Korea, and analyzed the behavior patterns of tourists from 2016 to 2017. Through statistical analysis of the behavior patterns of tourists, he proposed an optimal Tour route recommendation system [7]. Charles-Edwards employed big data together with geographic location information in 
Flickr social media to analyze the tourist attraction of six Italian cities [8]. Based on the GPS trajectory data of the social photosharing website (Flickr) and the network platform (Wikiloc), Sullivan took the Teide National Park as the research area, revealed the travel network and spatial distribution characteristics of tourists, and analyzed the social network geotag data [9].

The research mentioned above has made a significant contribution to the development of the tourism industry, but there is no in-depth study on mobile cloud IoT computing so far, and there is also a problem of insufficient awareness of the current cultural industry. China is a largely agricultural country with a long history, with a vast agricultural area, a profound agricultural culture, and strong rural customs. The development of rural leisure tourism has excellent conditions, strong demand, and broad prospects. In recent years, a lot of research has been conducted on the rural leisure tourism industry, but the research content mainly focuses on the competitiveness of the tourism industry, temporal and spatial distribution, market segmentation, influencing factors, and demand forecasting. Moreover, the existing research objects are relatively single, mainly limited to specific tourist destinations or tourist sources, and lack comprehensive systematic research.

The development of modern rural leisure tourism can provoke the readjustment of rural industrial structures. This is a powerful measure to achieve sustained, rapid, and healthy development of the rural economy. This is also of great significance for the sustainable development of society. However, rural leisure tourism data has not yet been effectively collected and processed. The existing limited tourism data is held by different agencies, such as tourism authorities, tourism data agencies, and cloud service providers. There is a lack of effective travel data exchange and sharing between these institutions. Therefore, various tourism data in different tourist areas should be safely collected, transmitted, and intelligently processed for overall decision-making and optimization analysis. All relevant government departments, agencies, and service providers must actively collaborate to meet the needs of cross-departmental data sharing in rural leisure tourism and ensure the security of user data and information. At the same time, rural leisure tourism should be constructed as a whole with the development of the modern rural economy, not just applied separately. If the modern rural economy is regarded as an ecosystem, rural leisure tourism should become an important link in this ecosystem.

This paper endeavors to build an intelligent and comprehensive application of the IoT for modern rural leisure tourism. A large number of IoT devices (such as various sensors, cameras, and cell phones) are used to monitor and collect various travel data and, then, aggregate them to the mobilecloud IoT computing platform. We use cloud computing and machine learning to intelligently analyze and optimize tourism data and obtain the best decision-making results overall. We take the Chinese rural leisure tourism industry as the analysis object, introduce the tourism market based on cloud computing, and analyze foreign tourism data to enhance the diversity of rural culture. This work also highlights the security requirements of government cloud data management and studies the measures to achieve cloud tourism data security.
The rest of the paper is organized as follows. Section II presents the mobile cloud IoT computing platform for rural leisure tourism. Section III develops the machine learning algorithm to mine the tourism data collected. Section IV uses China's rural leisure tourism sector as an example to examine the features of the national tourist market, followed by Section $\mathrm{V}$ to conclude the paper.

\section{Rural Leisure Tourism on Mobile Cloud IoT Computing Platform}

Mobile Cloud IoT Computing has great limitations in processing rural leisure tourism industry data. Although academia has done some exploration, the problems faced by the practice department are still difficult to solve. The reason is that the existing research strategies are relatively scattered, and the tourism data cloud security is not systematically constructed as an organic whole, and the interaction between the elements of the security system is not explored relationship [10]. Most of the existing strategies start from a single perspective of tourism data institutions or cloud service providers and ignore the coordination mechanism among different entities such as tourism data institutions, cloud service providers, and government cloud management units. Existing studies generally discuss "cloud", and usually point to the public cloud environment and fail to make a detailed analysis of the government cloud environment nor distinguish the different scenarios of tourism data institutions using government cloud services [11]. The core concept of the rural leisure tourism industry is conducive to understanding the symbiotic evolution relationship between different subjects in the cloud of tourism data and the external environment such as institutional environment, institutional environment, and market environment. It has reference value for proposing a more systematic security strategy of the tourism data cloud. Therefore, this paper introduces the perspective of the ecosystem to explore the security of tourism data systems [12].

We take the national cloud computing guidance tourism market as the research subject. For the availability of data, in the specific analysis of the characteristics of the national tourist market, this paper carries out a semistructured research on the heads of 11 tourism data institutions in Beijing, Shandong, Jiangsu, Henan, Inner Mongolia, and other regions, so as to understand the construction status and security needs of the tourism data management system in the government cloud.

On the basis of the traditional rural leisure tourism industry, the cultural mode of the original tourism area is retained, and the data analysis and integration guidance of Mobile Cloud IoT Computing Internet of things are added to guide tourists. The data integration model is dominated by tourism data institutions, and the data is transmitted to tourists by the server through cloud computing.

As shown in Figure 1, the first scenario is that the tourism data organization (including the tourism data center and the agency tourism data room) migrates the completed tourism data management system from the local to the government cloud or builds a new tourism data management system based on the government cloud (the system is only for the internal use of the tourism data organization). The 


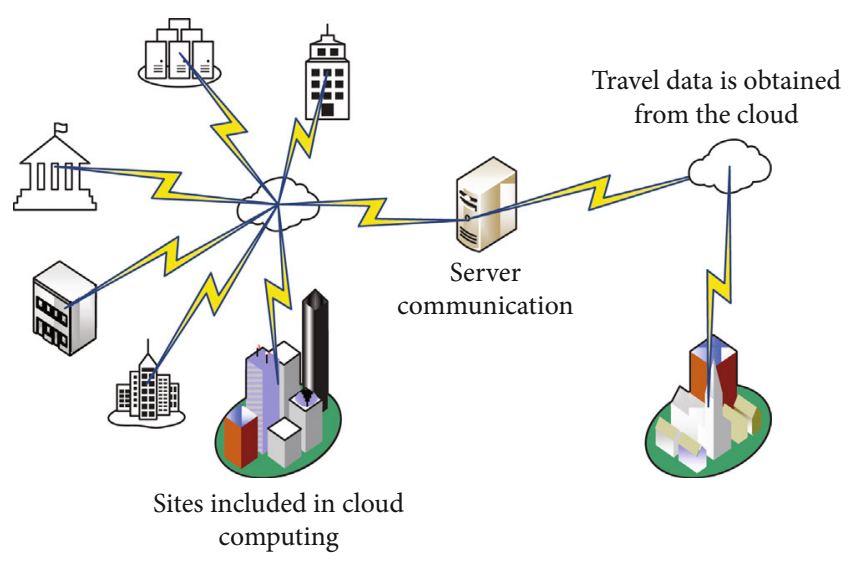

Figure 1: Cloud computing provides urban tourism data computing services.

second scenario is the construction of a unified tourism data management system based on government cloud by a regional comprehensive tourism data library under the organization and coordination of the tourism data authorities at the same level, including the tourism data management system for filing units and the digital tourism data library system for tourism data library, which provides the same level authorities' tourism data room and other comprehensive tourism data in the region. In different scenarios, producers, consumers, and decomposers refer to slightly different subjects. Based on the ecological model of tourism data cloud security at the macrolevel, this paper puts forward the security strategy for the tourism data security in the government cloud environment.

Based on the public cloud analysis model, this paper constructs the configuration model of the public cloud planning layout, which promotes the optimal allocation of public cloud resources under the objectives and constraints of service level agreement. Three types of resource pools are deployed according to different requirements of business scenarios: low-cost centralized deployment mode is adopted for cold scenic spot data and warm scenic spot data business to reduce cost and improve efficiency; in hot scenic spot data scenario, regional centralized deployment mode is adopted for high-density business and business side deployment mode is adopted for low-density business to ensure service quality according to the different business density of each region. At first, the public cloud is mainly used in telecom services, and the purpose is to create a healthy network ecological environment, so that tourists can enjoy better services and protect the rights and interests of tourists. Facing the practical problems in the planning of public cloud, public cloud analysis provides the idea of transmitting the business side service commitment to the back-end resource allocation ability, so that the business and resources can be configured simultaneously and effectively support the development of public cloud business.

\subsection{Demand Division of Cloud Computing in Tourism Planning} Calculus. Passenger flow is primarily focused in summer and autumn due to apparent seasonal variations in regional passenger flow, and there is an inadequate supply of tourist goods in spring and winter. By introducing four-season tourist goods, the tourism management department should take the appropriate steps to minimize the difference in tourism seasons and scientifically divert, steer, and control passenger flow. First and foremost, we will organize a regional ice and snow festival with the theme "Ice and Snow Outside the Great Wall, Silk Road Wonderland," and promote desert ice and snow tourism as a new winter and tourist business card. The size and appeal of ice and snow tourist goods are enhanced by spring tourism [13]. Northern China has released a succession of preferential policies, preferential policies, and subsidized policies for winter and spring tourism goods in recent years, but they have not boosted the market's vitality and are not favorable to tourist diversion flow. Diverse cultural tourist events will be organized at various peak periods in the future to bridge the gap between low and high seasons [14]. Increase participatory tourism experience activities incorporating historical and cultural elements, expand the play space and stay time of tourists in the region, and promote the West by creating RV campgrounds, customs outside the customs, or desert theme hotels, scientifically arrange certain tourist reception service facilities, increase participatory tourism experience activities incorporating historical and cultural elements, expand the play space and stay time of tourists in the region, and promote the West by creating RV campgrounds, customs outside the customs, or desert theme hotels. The route changes from tourist goods to participation experience tourism products, strengthening western route tourism products and decreasing the regional tourism flow's "core-periphery" hierarchical structure [15]. Taking the construction of the National Tourism Demonstration Zone as an opportunity, we will realize the efficient reorganization of tourism products and routes on the east-west line and further strengthen the construction of the transportation network to form the overall effect of tourism products. Take the western line passenger flow as an example, use the new media big data platform to promote the effective management of the tourist destination space and enhance the travel accessibility and radiation effect [16]. To promote effective management of tourist administration departments, use big data, new media, and smart tourism as platforms, it is feasible to disclose the visitor flow trajectory and tourist behavior preferences with high accuracy using tourist data information, to scientifically design tourist routes, to encourage efficient and precise destination marketing, and to further increase the potential tourist market. Promote the safe and healthy growth of the tourism sector in order to minimize the strain on visitor flow, particularly during the peak season of World Heritage tourism.

For cloud service providers, centralized deployment and decentralized deployment are indispensable. Telecom Tianyi cloud adopts the typical "centralized + decentralized" mode, but it is difficult to determine the matching principle of the two types of resource pools in the actual planning. Due to the utility characteristics of cloud, cloud tourists and cloud resource environment will constantly change. How to plan, schedule, and adjust cloud resources in a dynamic environment to meet the complex needs of tourists has become an urgent problem in a cloud computing environment. Therefore, cloud resource layout planning and design must evaluate business needs, predict business development trends and adjust the layout, in order to balance operating costs and 
customer perception, and occupy a favorable position in the competition. The significance of service level agreement (SLA) between cloud tourists and cloud service providers is not only to protect the rights and interests of customers but also to reveal the needs of tourists to service providers. How to transmit the business side service commitment to the back-end resource allocation strategy and promote the optimal allocation of cloud resources under the objectives and constraints of SLA is the key to solve the problem of cloud resource allocation. It is an effective way to solve the problem of the source distribution. Based on the different needs of tourists, business scenarios are different resources are configured to maximize the satisfaction of tourists and resource utilization of cloud service providers. According to the different needs of tourists, public cloud business scenarios are divided into three categories: cold, hot, and warm. For typical industries and key products, cloud business scenarios are classified, and different resource requirements (delay, performance, etc.) of SLA levels are formulated.

\section{Machine Learning-Based Tourism Data Analysis}

3.1. Tourism Data Security Based on Machine Learning. To meeting the requirement of tourism data protection, this paper explores the security cooperation mechanism between tourism data authorities and tourism data preservation institutions. Since 2018, most of the local tourism bureaus have been actively reformed to set up their own data centers. Some interviewees said that after the reform, the competent departments of tourism data perform the administrative functions and do not assume the responsibility of preserving tourism data [17]. There is no strong demand for tourism data management based on the government cloud. After the institutional reform, the competent departments of tourism data are generally short of manpower, but they have to undertake the functions of four or five departments, including professional title evaluation, education and training, law enforcement, and inspection [18]. In addition, most of the staff do not have a professional background in tourism data science or working experience in tourism data, so they have a negative attitude towards the security risks and avoidance strategies of tourism data in the government cloud environment [19]. In this case, the competent department of tourism data should strengthen the cooperation and contact with the tourism data center and the agency tourism data room, reach an agreement on the security issues of the application of government cloud by tourism data institutions, and strengthen the cooperation [20].

We will improve the legal system, standards, and norms and promote pilot projects. Based on the opinions, on the one hand, the competent departments of tourism data should timely issue operational system specifications to guide the risk identification and assessment of tourism data institutions and provide reference framework and methodological support for the formulation of risk response strategies; on the other hand, according to the superposition of security needs in the political cloud environment, they should fully implement the system specifications of tourism data security considering the security risks brought by the government cloud, and this paper proposes and refines the tourism data security requirements applicable to the government cloud environment [21]. The concept of superposition of security requirements is not a unique product in the cloud environment, but the traditional informatization [22].

Through application review, expert review, and third review, the tourist data authority can advise the comprehensive tourism data center and government tourism data room on how to choose system developers and cloud service agents. Improve the quality of service provided by tourist data information technology and content management software businesses, as well as the provision of tourism data information-related systems, platforms, tools, and other goods. Many respondents indicated that localized goods are not cost-effective, especially in terms of localized replacement, and that there is a conflict between safety and costeffectiveness. Based on attaining safety, dependability, and self-control, relevant firms should enhance the functional integrity and performance stability of their goods [23].

3.2. Interactive Mechanism Model of Tourism Data. Aiming at the innovation and development of the tourism rural leisure tourism industry, this paper constructs the interactive mechanism model of tourism data subject combined with cloud computing [24]. Tourism data institutions, cloud service providers, and government cloud management units are all important components of tourism data cloud security ecology, and the construction of subject interaction mechanism needs Mobile Cloud IoT Computing [25]. Among them, the Internet is the key, focusing on clear security responsibilities and responsibility boundaries; cloud computing is the foundation, focusing on communication and cooperation among the three; supervision and audit is an important way to build a new relationship among them, and let $n$ be the number of boundary points of cloud computing, then the relationship strength $G$ can be expressed as

$$
\begin{gathered}
G=\frac{\sum_{Z=1}^{h_{j}} \sum_{r=1}^{n_{h}}\left|y_{j i}-y_{h r}\right|}{n_{j} n_{h}\left(u_{j}+u_{h}\right)}, \\
l=1-\frac{\left(2 \mu_{x} \mu_{y}+C_{1}\right)\left(2 \sigma_{x y}+C_{2}\right)}{\left(\mu_{x}^{2}+\mu_{y}^{2}+C_{1}\right)\left(\sigma_{x}^{2}+\sigma_{y}^{2}+C_{2}\right)} .
\end{gathered}
$$

Among them, $n$ represents the annual increase in the number of visitors in the society, $u$ is the number of visitors affected by the Internet, $Z$ is the counting unit, and $h$ is the peak value of visitors. In the formula (2), the subscript of $u$ is the visitor integral interval, and $C_{1}$ and $C_{2}$ are the scattered point identifications of tourists in the integral interval, $y$ is the end of the error, $\mathrm{n}$ is the number of nodes, and $\mu$ is the error representation of the number of visitors. In formula (2), $\sigma$ is a negative error coefficient, and $x$ and $y$ are space point elements, respectively, and $l$ is the degree of spatial relationship. This paper mainly uses the theoretical perspective of the ecosystem to carry out research and applies it at the macro and micro levels. At the macrolevel, we use the 
natural ecosystem theory in the field of ecology for reference to build a tourism data security model. The following equations calculate the core index $(\wp)$ used to reflect the spatial concentration of cloud computing guiding the tourism market:

$$
\begin{gathered}
\wp_{\kappa}=\frac{2 k}{k+1}+\left[\frac{1}{2}+\frac{1}{2 k}\right]\left[\frac{c_{2}-c_{1}}{3}\right]^{2}+\frac{2\left(c_{2}-c_{1}\right)}{3}, \\
D_{j h}=\int_{0}^{\infty} d F_{j}(y) \int_{0}^{y}(y-x) d F_{h}(x), \\
G_{t}=\sum_{j=2}^{k} \sum_{h=1}^{j-1} G_{j h}\left(p_{j} s_{h}+p_{h} s_{j}\right) D_{j h}\left(1-D_{j h}\right),
\end{gathered}
$$

where $G$ is the number of tourists from the k country of origin, the meaning of $C$ in the formula is the same as formula (2), $t$ is the total number of inbound tourists from China in that year, and $N$ is the number of countries of origin. $F()$ is the tourist error function, and $d$ is the representation of the integral, where it represents the integral of $F()$. The above formula (5) expresses the relationship between $G$ and the integration result " $D_{j h}$ " of formula (4), $j, x$, and $y$ are the identifiers of the elements. The meaning of $G$ here is the same as formula (1). The greater the $G$ value, the more concentrated the spatial distribution of tourists, and the greater China's dependence on the market. It can be used to analyze the competition level and development potential of the major source countries in the cloud computing-guided tourism market. We further make the following definitions:

$$
\begin{aligned}
& f(x)=\frac{1}{N h} \sum_{i=1}^{N} k\left(\frac{X_{i}-x}{h}\right), \\
& k(x)=\frac{1}{\sqrt{2 \pi}} \exp \left(-\frac{x^{2}}{2}\right),
\end{aligned}
$$

where $f(x)$ and $N h$ represent the occupancy rate and the growth rate, $X_{i}-x$ is the first time that year $h$ number of tourists from each country of origin, $X_{i}$ is the number of tourists in the source country last year, and $N$ is the total number of Chinese Inbound Tourists in that year. The average values of $\alpha$ and $\beta$ in the period are calculated as $k(x)$, and the twodimensional coordinates are divided to obtain the competitive state of each customer market in the country.

In this work, we utilize a specific time series model, a method to predict the future by using past observations, which can effectively predict the development trend of national cloud computing to guide the tourism market. The general form is as follows:

$$
\begin{gathered}
\ln \left(\frac{x}{x-1}\right)=\beta \ln x-1, \\
\psi=\sum_{x=1}^{\theta} V x=\sum_{x=1}^{9}\left(\frac{W x}{\sum_{1}^{n} W_{\mathfrak{I}}} S x\right) .
\end{gathered}
$$

In formula (7), $x$ is the integral function and $\beta$ is the time coefficient. In formula (8), the meaning of $x$ is the same as formula (7), $W$ is the difficulty coefficient of conventional guidance, and $\theta$ is the service area of the tour guide. Grey correlation analysis is a method to evaluate the degree of correlation between various indicators, which can be used to analyze the impact of various factors on the national tourists' cloud computing guidance tourism demand. The steps are as follows: first, determine the reference sequence $h_{t}$ and comparison sequence $w_{c} x_{t}$. The relationship between them is as follows:

$$
\begin{gathered}
h_{t}=\tan h\left(w_{c} x_{t}+u_{c}\left(r_{t} \Theta h_{t-1}\right)+b_{c}\right), \\
h_{t}=z_{t} \Theta h_{t-1}+\left(1-z_{t}\right) \Theta h_{t} .
\end{gathered}
$$

In formula (10), $z$ is the coefficient of determination, corresponding to $h, t$ is the increment of time, and $\Theta$ is the direction conversion of the vector to maintain the validity of the result $h_{t}$.

Second, the data are dimensionless, in formula (11), $L$ represents the complex coefficient of curve processing, and $p$ and $q$, respectively, represent the value of the trigonometric function mapping relationship in the prediction.

$$
\theta(p, q)=\arctan \left(\frac{L(p, q+1)-L(p, q-1)}{L(p+1, q)-L(p-1, q)}\right)
$$

Finally, the correlation coefficient and correlation degree are calculated in the following:

$$
\begin{gathered}
\ln \left(\frac{F I_{i t}}{F I_{i t}-1}\right)=\alpha+\beta \ln F I_{i t}-1, \\
k_{t 1}[i]=\sum_{j} \cos \left(w_{i}^{1}, w_{j}^{2}\right) .
\end{gathered}
$$

Among them, $\alpha$ is the coefficient of rural tourism in the previous year, $\beta$ is the coefficient predicted this year, and $i$ and $j$ indicate the difficulty coefficient of unlimited tempering. The degree of grey correlation FI represents the influence of cloud computing guided tour demand, which has a positive correlation.

\section{Numerical Results and Analysis}

4.1. Tourism Data Analysis with Mobile Cloud IoT Computing. Using the formula of entropy method to calculate the rural tourism development potential and its subsystem index of county unit in the region, the calculation results are shown in Table 1.

The measurement results are shown in Figure 2. The regional rural tourism development potential index is the highest (0.572), which is about 5 times of the lowest. Further statistics show that the overall development potential of rural tourism in the region is relatively low and the difference is obvious, among which $59.09 \%$ of the counties and districts have lower development potential than the average. Comparing the distribution of the development index of the 
Table 1: Rural Tourism Development Potential and Its Subsystem Index.

\begin{tabular}{lccccc}
\hline Item & NSSP & Strategy & Government affairs & Cloud environment & Legal system \\
\hline Inbound & 1.15 & 1.83 & 0.52 & 0.62 & 2.26 \\
Visitors & 3.97 & 1.21 & 3.49 & 3.37 & 0.76 \\
Geography & 3.85 & 2.03 & 3.6 & 4.44 & 5.47 \\
Concentration & 2.83 & 2.66 & 2.83 & 3.61 & 5.9 \\
Specification & 1.49 & 3.08 & 4.17 & 4.09 & 5.92 \\
Project pilot & 2.82 & 6.56 & 1.11 & 1.51 & 1.42 \\
Market & 5.41 & 2.76 & & & \\
\hline
\end{tabular}

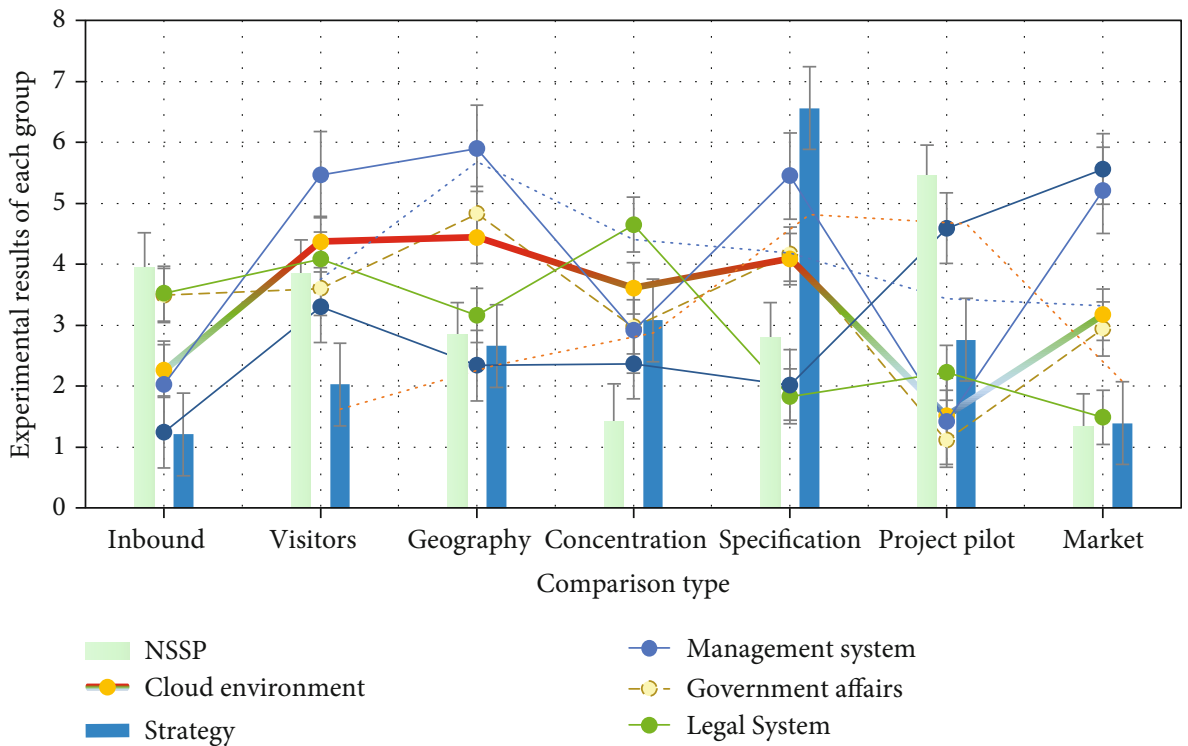

FIGURE 2: Regional rural tourism development potential index.

five subsystems, we can see that the conditional coefficient of variation of rural tourism elements is the largest, followed by the economic support of rural tourism and the tourism industry foundation of rural tourism region, while the endogenous development level of rural tourism is relatively small, and the variation coefficient of rural tourism environment background and rural tourism development potential is relatively close.

As shown in Figure 3, about 5.6\% of the high-level rural tourism development potential areas are in the district and county units. Most of these areas are located in cities and autonomous prefectures divided into districts, which have a good tourist market environment and regional economic development foundation. The development potential index of rural tourism is more than 0.5 . It is found that the high development potential of rural tourism is mainly due to the outstanding performance of A-level scenic spots, the number of traditional villages, and the number of starrated farmhouse and star-rated rural tourism inns. The improvement of rural tourism reception capacity and supporting service facilities in this grade area provides a good foundation for the development of rural tourism.

As shown in Figure 4, the industrial base of rural tourism, the number of regional tourists and tourism economic income, high star hotels, and other indicators also show obvious advantages, providing strong competitiveness for the development of rural tourism. Further exploring the elements of each subsystem, it is found that the background index of rural tourism environment in high-level development potential areas is generally low, which also restricts the further improvement of rural tourism development potential in this area to a certain extent.

The security of tourism data in e-government cloud environment is a complex system engineering, involving many subjects and elements. However, most of the existing literature tends to put forward suggestions and requirements for tourism data institutions and cloud service providers, respectively, ignoring the relevance. Therefore, based on the theoretical framework of the natural ecosystem and the results of cloud computing coding, this paper constructs a macrolevel ecological model of tourism data cloud security. The model focuses on the interaction between the subject and the environment and the interaction between the subjects. The security environment includes a management system, legal system, standards, project pilot, market guidance, and other specific environments. The security subject refers to the organization that undertakes the responsibility of tourism data security and can understand the tourism data 


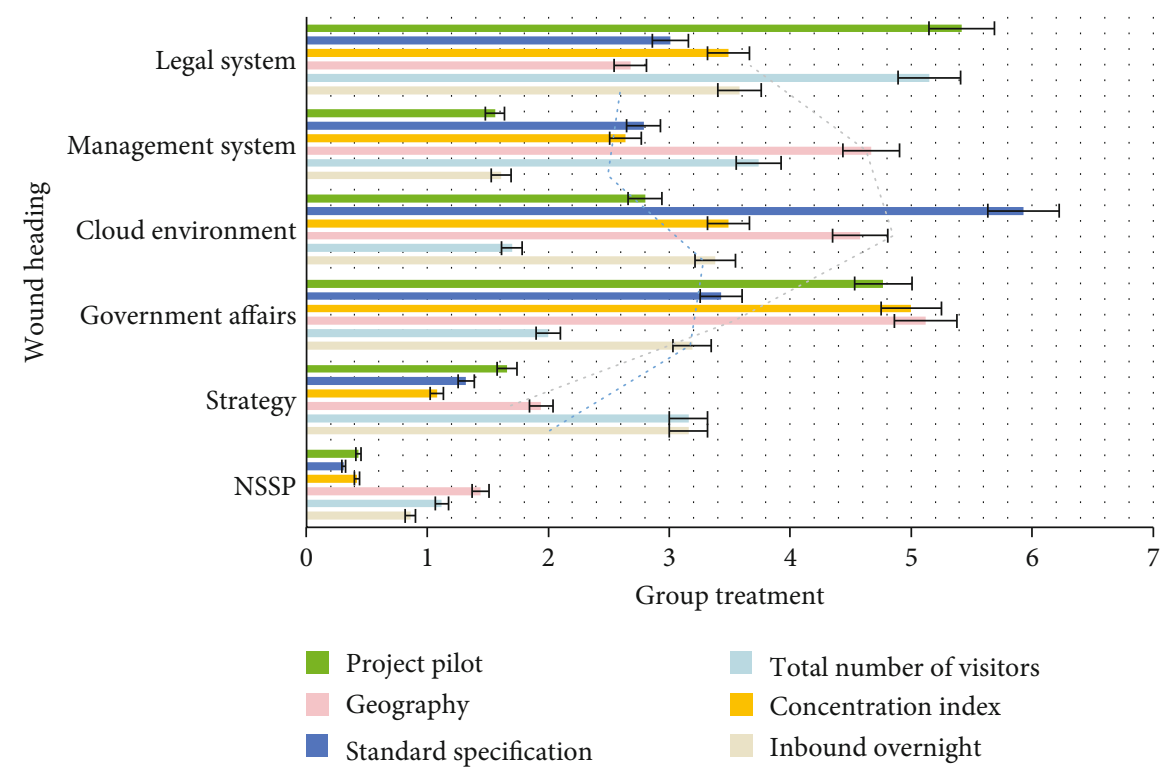

FIGURE 3: High-level rural tourism development potential.

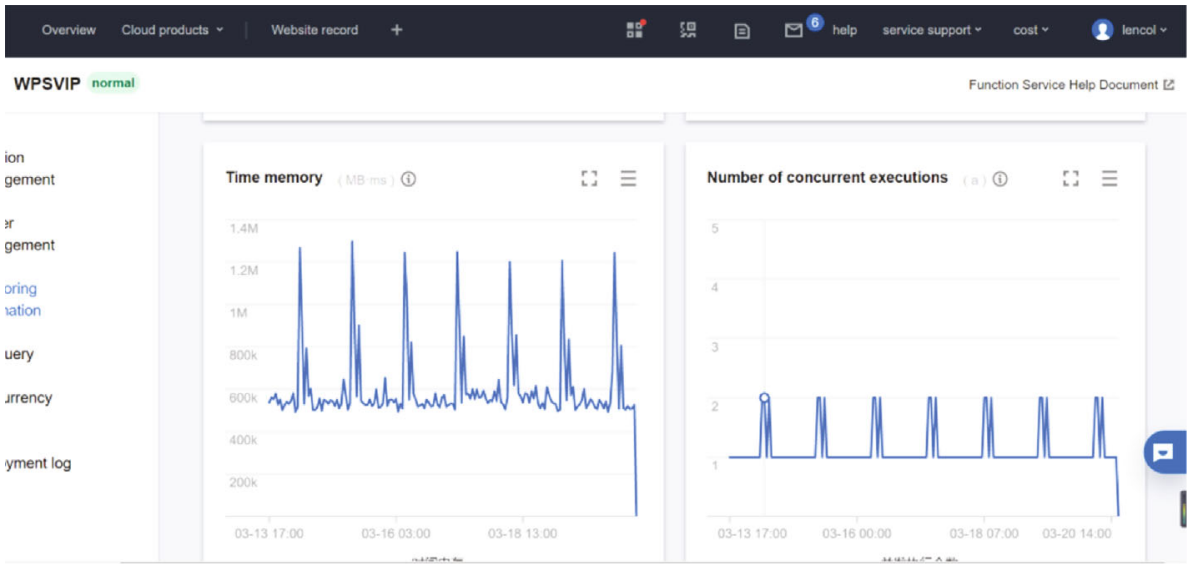

FIgURE 4: Tencent Cloud's tourism data cloud computing data statistics chart (Statistics services provided by Tencent Cloudhttps://console .cloud.tencent.com/).

cloud security visually by producers, consumers, and decomposers. It also distinguishes the two kinds of tourism data in the cloud data Table 2.

As shown in Figure 5, the seasonal difference of regional passenger flow is obvious, and the regional passenger flow is mainly concentrated in summer and autumn. Generally speaking, the regional passenger flow presents the seasonal variation law. To explore the different characteristics, this study introduces the seasonal index and climate comprehensive comfort (CCI). The results show that the seasonality index of regional tourist flow tends to be 11.218 in recent years, and the seasonality index is too large and shows a stable trend in three years, which indicates that the seasonality difference of regional tourist flow presents a continuous solidification trend.

As shown in Table 3, the development potential of rural tourism in the region as a whole presents the characteristics of "center periphery" spatial structure. The high level of rural tourism is concentrated in the provincial and municipal central city and its surrounding areas, while the low level of rural tourism development potential is scattered and far away from the provincial and municipal central city. However, the low-level radiation driving effect of high-level rural tourism development potential on the surrounding areas is not significant, and the overall regional linkage development and regional cooperation are not significant. It needs to be strengthened.

As shown in Figure 6, since the formal signing of the National Free Trade Zone framework, the number of national cloud computing guided tourism has been growing, reaching new heights. In terms of quantity, the total number of national tourists, inbound overnight tourists, and inbound foreign tourists in China is on the increase; in terms of proportion, the proportion of national tourists in the total number of 
TABLE 2: Two scenarios of travel data to the cloud.

\begin{tabular}{lccccc}
\hline Item & Inbound overnight & Geography & Concentration index & Standard specification & Project pilot \\
\hline NSSP & 1.54 & 1.33 & 1.55 & 1.7 & 2.84 \\
Strategy & 2.22 & 3.22 & 2.04 & 4.52 & 2.31 \\
Government & 4.23 & 2.66 & 5.13 & 4.66 & 3.48 \\
Management system & 2.54 & 1.35 & 5.29 & 1.31 & 4.64 \\
Legal system & 2.25 & 2.01 & 2.89 & 1.31 \\
\hline
\end{tabular}

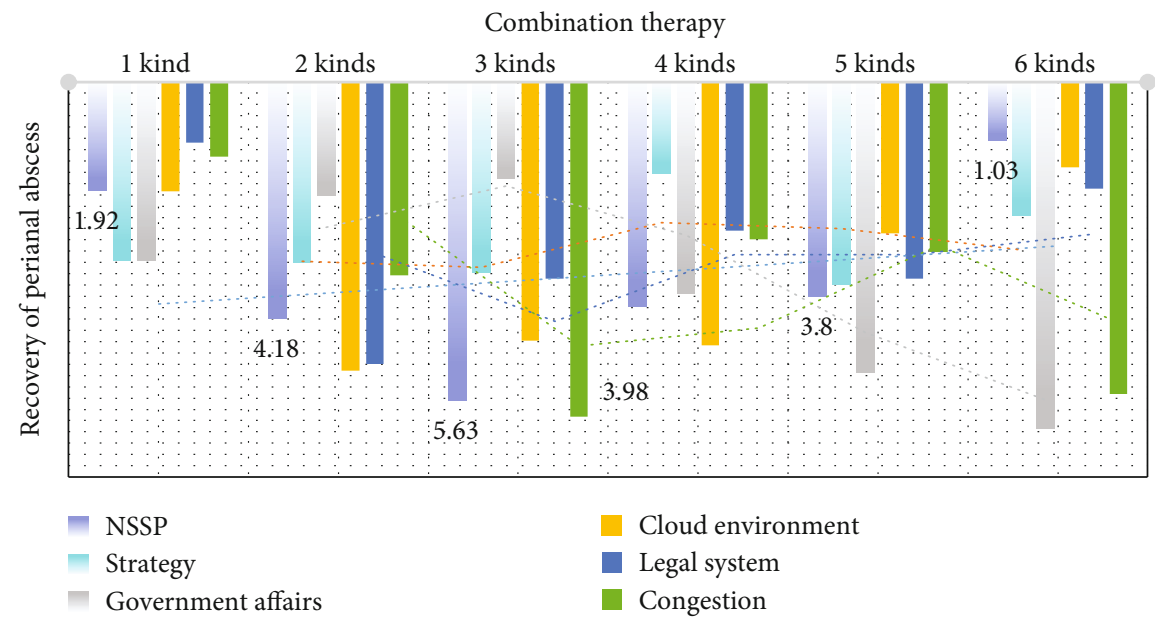

Figure 5: The seasonal difference of regional passenger flow is obvious.

TABLE 3: Regional rural tourism development potential.

\begin{tabular}{lccccc}
\hline Item & Management & Standard & Specification & Legal system & Market guidance \\
\hline Center & 1.44 & 0.5 & 0.61 & 1.35 & 1.67 \\
Peripheral & 1.37 & 2.91 & 1.93 & 4.55 & 2.71 \\
NSSP & 3.87 & 2.78 & 2.61 & 1.75 & 3.78 \\
Strategy & 3.94 & 1.69 & 3.34 & 3.93 & 2.48 \\
Government & 2.15 & 4.87 & 4.68 & 4.76 \\
\hline
\end{tabular}

inbound overnight tourists and inbound foreign tourists is on a steady growth trend.

The geographical concentration index and share of the national cloud computing guided tourism market draw an interannual change chart, as shown in Figure 7. The geographical concentration index $G$ of the main tourist source markets in China shows a continuous increasing trend, indicating that China's foreign tourist source countries are more and more concentrated in geographical distribution. The value of $G$ is between 0.9 and 2.33, and the degree of dependence on the national tourist market is relatively small. After 2019 , the $G$ value will rise to 9.60 in 2021, and China's dependence on the national tourist market will also increase significantly. It can be seen that the status of the whole country in China's rural leisure tourism market is increasingly important.

As shown in Figure 8, it is predicted that the cloud computing guided tourism demand of both the whole country and the major source countries will basically maintain a sustained and stable growth trend, and the market development prospect is broad. In general, China is expected to receive 25.5799 million tourists from all over the country in 2026, far more than the total number of Asian inbound tourists (19.1207 million) in 2020, and it will remain at more than 25 million every year since then; among the seven major source countries, each source country has maintained a steady growth year by year. Therefore, with the increasingly close ties between China and the whole country, it can be predicted that the potential of the national tourist market is huge. In the future, it will not only continue to consolidate its market position as a major tourist source in Asia but also become a key area for the development of the rural leisure tourism market in China.

As shown in Figure 9, after ranking the correlation degree values, it is found that the influence degree of each factor on the national cloud computing guided tourism is 


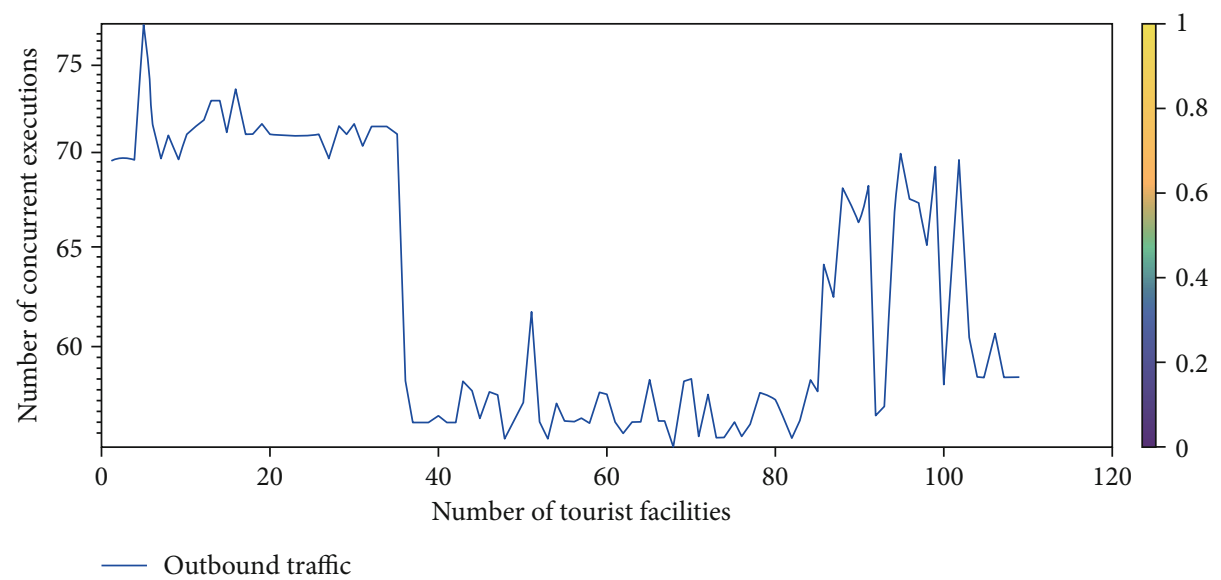

FIGURE 6: Cloud computing guides travel needs.

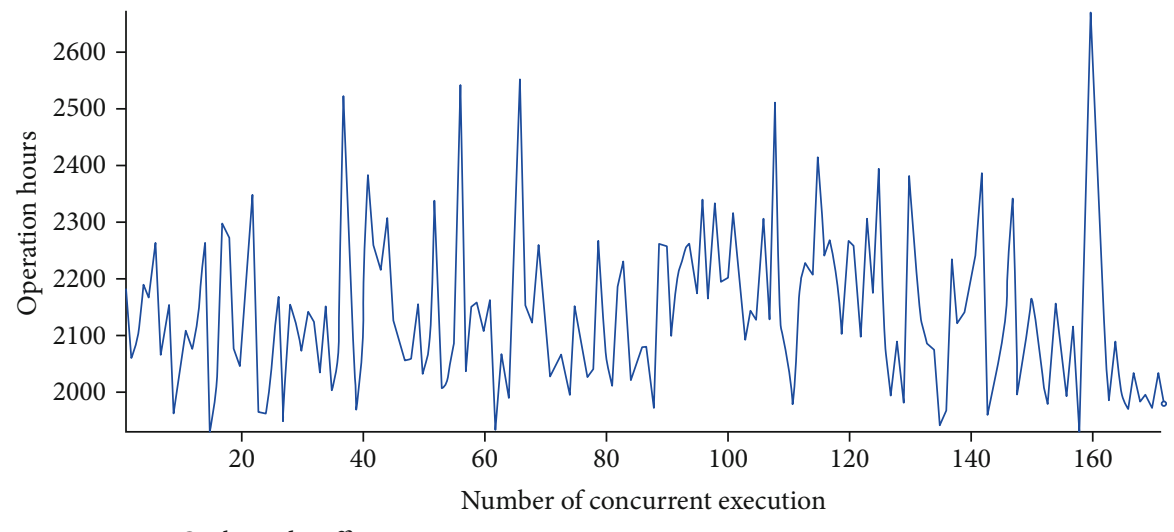

O Outbound traffic

Figure 7: Cloud computing guides the number of people traveling.

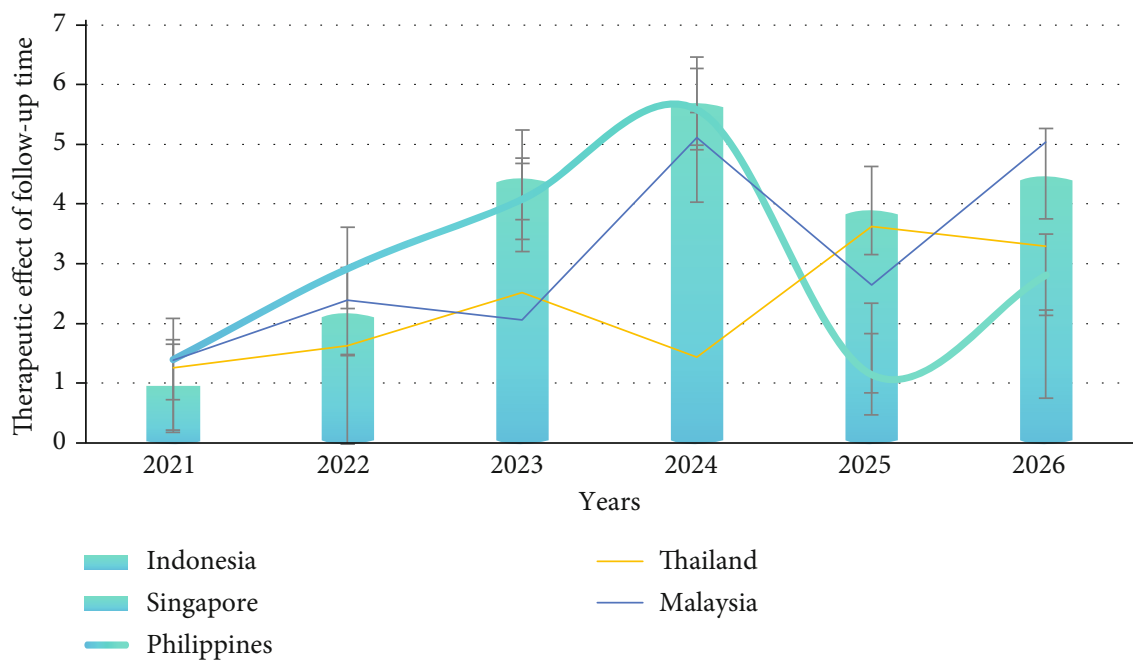

Figure 8: Cloud computing guides travel needs. 

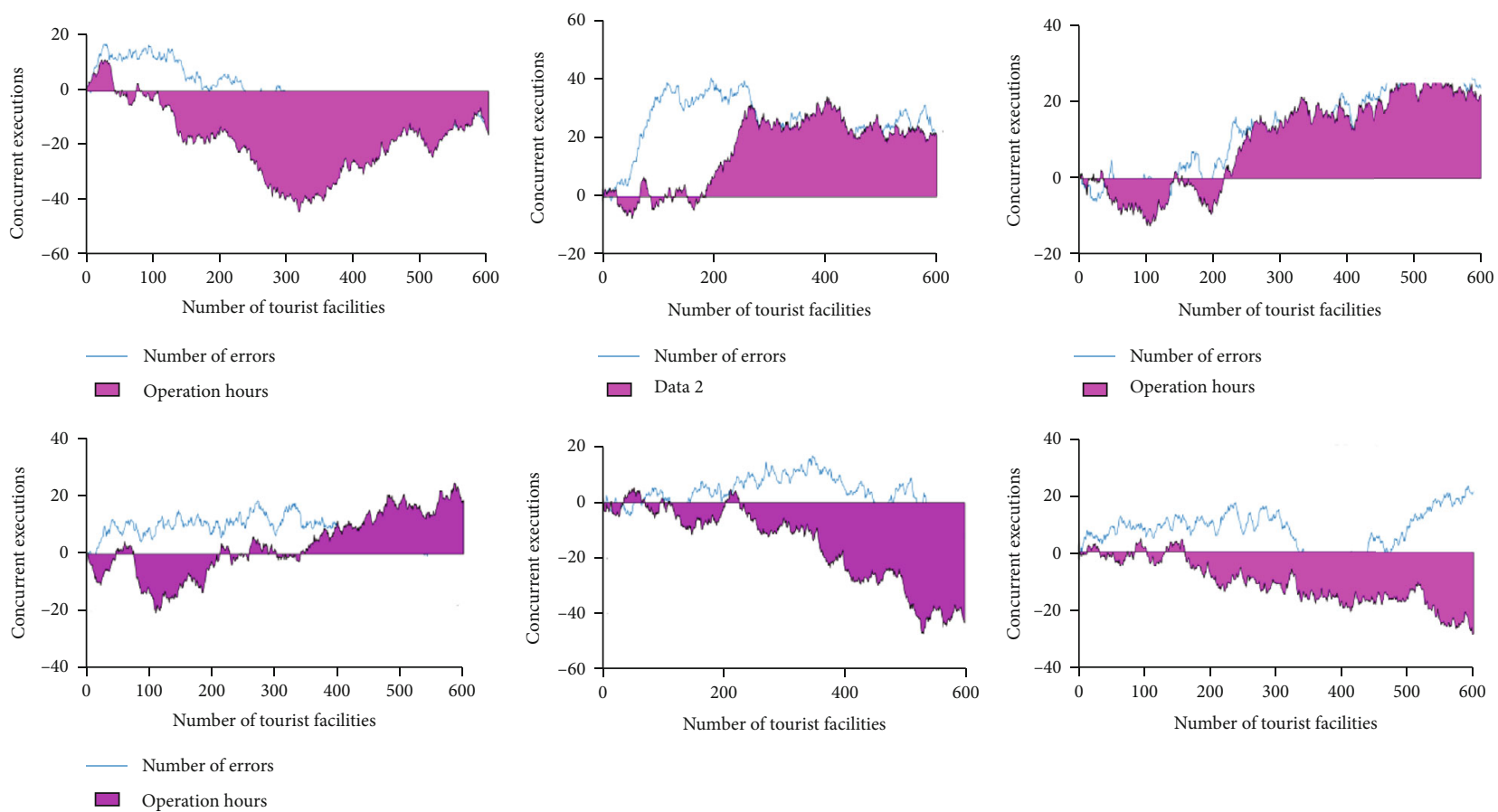

FIgURE 9: National cloud computing guide tourism market.

as follows: international route transportation mileage (0.832), bilateral trade volume $(0.812)$, China's per capita GDP (0.805), tourism reception facilities (0.778), and the number of world heritage sites (0.765). Among them, the international route transportation mileage $(\times 4)$ has the largest correlation, which indicates that for the national tourists, the tourism transportation convenience, especially the shipping conditions, has the most profound impact on their enthusiasm and demand in China. The correlation degree of bilateral trade volume $(\times 5)$ takes the second place, which shows that under the background of globalization, the driving role of international trade in tourism cannot be underestimated. The higher the level of economic exchanges in China, the more effective it is to drive the demand of tourists to China.

4.2. Findings and Discussions. The statistical method of cloud computing is used to investigate the characteristics, development trend, and influencing variables of the national cloud computing tour guide industry. In terms of market development, the national cloud tour guide market exhibits an overall stable, continuous, and positive growth trend. The overall number of Chinese tourists is increasing, and rural leisure tourism in China has shifted to the national region. Rural leisure tourism is a significant component of the tourism industry. It has a general homogeneity and individual concentration in its temporal distribution, and the regional distribution of the interyear concentration index shows an increasing concentration trend. From 2019, the geographic concentration index begins to rise linearly, peaking at 9.60 in 2020.

At the same time, despite changes in country share rankings, market concentration is still high and dependence on a few major source countries is too strong. We should take advantage of the "One Belt, One Road" initiative and China-FTA platform to strengthen mutual trust, cultural exchanges, and economic cooperation, accelerate the implementation of mutual visa-free tourism policy, promote tourism exchanges and cooperation, and improve the level of Mandarin reception to attract more tourists from all over the country. On the other hand, differentiated development should be carried out for various types of visitor source markets. The correlation between China's GDP per capita and resident rural leisure tourism is in the middle of the range, with no significant difference from the first two indicators, indicating that national economic development is closely related to resident rural leisure tourism, and national travelers are more willing to go to countries with better economic development. Domestic travelers are concerned about China's tourism resource endowment and tourism reception capacity, as the correlation between the number of tourism reception facilities and the number of world cultural heritage sites is also greater than 0.75 .

\section{Conclusions}

The growth potential of rural tourism has shown apparent geographical autocorrelation and spatial agglomeration in the mobile cloud IoT computing environment. The counties with the most potential for rural tourist growth, as well as those with the least, exhibit geographical clustering patterns. The province's high-level rural tourist development potential is concentrated in the center, whereas the province's low-level rural tourism development potential is dispersed across the province, city, and district. The radiation impact of high-level regions on nearby low-level growth potential 
areas, on the other hand, is minimal, and overall regional linkage development and regional collaboration must be increased. The growth potential of rural tourism in the mobile cloud IoT computing environment is unequal, and the difference is mostly due to the abundance of rural tourism resources and the degree of economic development.

\section{Data Availability}

All the data used is given in the paper.

\section{Conflicts of Interest}

The authors declare that they have no conflicts of interest.

\section{References}

[1] X. Chen, L. Jiao, W. Li, and X. Fu, "Efficient multi-user computation offloading for mobile-edge cloud computing," IEEE/ACM Transactions on Networking, vol. 24, no. 5, pp. 2795-2808, 2016.

[2] Y. Dai, D. Xu, S. Maharjan, and Y. Zhang, "Joint computation offloading and user association in multi-task mobile edge computing," IEEE Transactions on Vehicular Technology, vol. 67, no. 12, pp. 12313-12325, 2018.

[3] Y. Zhou, L. Tian, L. Liu, and Y. Qi, “Fog computing enabled future mobile communication networks: a convergence of communication and computing," IEEE Communications Magazine, vol. 57, no. 5, pp. 20-27, 2019.

[4] Y. Qi, L. Tian, Y. Zhou, and J. Yuan, "Mobile edge computingassisted admission control in vehicular networks: the convergence of communication and computation," IEEE Vehicular Technology Magazine, vol. 14, no. 1, pp. 37-44, 2019.

[5] Y. Zhou, L. Liu, L. Wang et al., "Service-aware 6G: an intelligent and open network based on the convergence of communication, computing and caching," Digital Communications and Networks, vol. 6, no. 3, pp. 253-260, 2020.

[6] P. Porambage, J. Okwuibe, M. Liyanage, M. Ylianttila, and T. Taleb, "Survey on multi-access edge computing for internet of things realization," IEEE Communications Surveys \& Tutorials, vol. 20, no. 4, pp. 2961-2991, 2018.

[7] S. K. Smith and M. House, "Snowbirds, sunbirds, and stayers: seasonal migration of elderly adults in Florida," The Journals of Gerontology Series B: Psychological Sciences and Social Sciences, vol. 61, no. 5, pp. S232-S239, 2006.

[8] E. Charles-Edwards and M. Bell, "Seasonal flux in Australia's population geography: linking space and time," Population Space and Place, vol. 21, no. 2, pp. 103-123, 2015.

[9] D. A. Sullivan and S. A. Stevens, "Snowbirds," Research on Ageing, vol. 4, no. 2, pp. 159-177, 1982.

[10] T. D. Hogan, "Determinants of the seasonal migration of the elderly to sunbelt states," Research on Ageing, vol. 9, no. 1, pp. 115-133, 1987.

[11] J. Silaa, H. Jazri, and H. Muyingi, "A study on the use of mobile computing technologies for improving the mobility of Windhoek residents," African Journal of Science Technology Innovation and Development, vol. 9, no. 1, pp. 1-15, 2021.

[12] Y. Li, K. Kim, and Y. Ding, "Early warning system of tennis sports injury risk based on mobile computing," Mobile Information Systems, vol. 2021, Article ID 3278276, 10 pages, 2021.
[13] J. Zhang, X. Hu, Z. Ning et al., "Energy-latency tradeoff for energy-aware offloading in mobile edge computing networks," IEEE Internet of Things Journal, vol. 5, no. 4, pp. 2633-2645, 2018.

[14] F. Wang, J. Xu, X. Wang, and S. Cui, "Joint offloading and computing optimization in wireless powered mobile-edge computing systems," IEEE Transactions on Wireless Communications, vol. 17, no. 3, pp. 1784-1797, 2018.

[15] Y. Ding, C. Liu, X. Zhou, Z. Liu, and Z. Tang, "A code-oriented partitioning computation offloading strategy for multiple users and multiple mobile edge computing servers," IEEE Transactions on Industrial Informatics, vol. 16, no. 7, pp. 4800-4810, 2020.

[16] G. Zhefeng, J. Wang, J. Congfeng et al., “Analysis of resource utilization of co-located clusters," Chinese Journal of Computers, vol. 43, no. 6, pp. 1103-1122, 2020.

[17] C. Xu, X. Xiong, Q. Du et al., "Dynamic scheduling model of rail-guided vehicle (RGV) based on genetic algorithms in the context of mobile computing," International Journal of Mobile Computing and Multimedia Communications, vol. 12, no. 1, pp. 43-62, 2021.

[18] A. Alkhalil, "Evolution of existing software to mobile computing platforms: framework support and case study," International Journal of Advanced and Applied Sciences, vol. 8, no. 3, pp. 100-111, 2021.

[19] Y. Hmimz, T. Chanyour, M. el Ghmary, and M. O. Cherkaoui Malki, "Joint radio and local resources optimization for tasks offloading with priority in a mobile edge computing network," Pervasive and Mobile Computing, vol. 73, no. 1, article 101368, 2021.

[20] J. He, D. Zhang, Y. Zhou, and Y. Zhang, "A truthful online mechanism for collaborative computation offloading in mobile edge computing," IEEE Transactions on Industrial Informatics, vol. 16, no. 7, pp. 4832-4841, 2020.

[21] C. Wang, C. Liang, F. R. Yu, Q. Chen, and L. Tang, "Computation offloading and resource allocation in wireless cellular networks with mobile edge computing," IEEE Transactions on Wireless Communications, vol. 16, no. 8, pp. 4924-4938, 2017.

[22] T. Wang, M. Z. A. Bhuiyan, G. Wang, L. Qi, J. Wu, and T. Hayajneh, "Preserving balance between privacy and data integrity in edge-assisted internet of things," IEEE Internet of Things Journal, vol. 7, no. 4, pp. 2679-2689, 2020.

[23] Y. Bowen, P. Lingjun, X. Yuting, X. Jingdong, and Z. Jianzhong, "Joint task offloading and base station association in mobile edge computing," Journal of Computer Research and Development, vol. 55, no. 3, pp. 537-550, 2018.

[24] Y. Wang, M. Sheng, X. Wang, L. Wang, and J. Li, "Mobile-edge computing: partial computation offloading using dynamic voltage scaling," IEEE Transactions on Communications, vol. 64, no. 10, pp. 4268-4282, 2016.

[25] T. Q. Dinh, "Offloading in mobile edge computing: task allocation and computational frequency scaling," IEEE Transactions on Communications, vol. 65, no. 8, pp. 3571-3584, 2017. 